A Review of the Interleaving Effect: Theories and Lessons for Future Research

\author{
Veronica X. Yan \\ Department of Educational Psychology \\ The University of Texas at Austin
}

\author{
Brendan A. Schuetze \\ Department of Educational Psychology \\ The University of Texas at Austin
}

Luke G. Eglington

Institute for Intelligent Systems

University of Memphis

Author note: Portions of this research were presented at the 60th annual meeting of the Psychonomic Society in Montreal, Canada. We thank Matthan Moy and Kimberly Nguyen for their assistance in coding.

Correspondence concerning this article should be addressed to Veronica X. Yan, Department of Educational Psychology, The University of Texas at Austin, 1912 Speedway STE 504, Austin TX 78712. Email: veronicayan@austin.utexas.edu 


\begin{abstract}
Numerous studies have shown that an interleaved study sequence of examples (e.g., $\mathrm{ABCBACACB}$ ) from different categories, relative to a blocked sequence (e.g., AAABBBCCC), often yields superior category learning. Some explanations for sequencing effects centers on attentional processes, others focus on memory processes, and the two are often pitted against each other. We propose a new integrative two-stage framework for sequencing effects in category-learning and support this framework using a meta-analytic approach. We show, using a meta-analytic approach, that a combination of memory and attentional predictors explains significantly more variance in sequencing effects than attentional factors alone. This approach also allowed us to examine the nature of the existing evidence, which revealed inferential limitations due to how researchers typically design experiments. We provide suggestions for future research on sequencing effects in category learning that would both test the two-stage framework and increase the impact of future research.
\end{abstract}

Keywords: interleaving, spacing, sequencing effects, category learning, theoretical framework 


\section{A Review of the Interleaving Effect: Theories and Lessons for Future Research}

In our everyday lives, the need to make rapid decisions is supported by our fundamental capacity to categorize the world around us (e.g., objects, people, experiences, actions). While there are many different approaches researchers have taken to examine what processes are involved in category learning, an emerging body of literature has focused on how the study and practice of multiple categories should be sequenced. This research has revealed a strikingly counterintuitive finding: instead of focusing on learning one category at a time (referred to as 'blocking'), it can be better to mix up and switch between different categories (referred to as 'interleaving'). In the present paper, we conduct both a critical review and a quantitative review of the interleaving effect in category learning. First, we review the existing theories, finding that they largely fall into one of two categories: those relating to attention and those relating to memory. Next, we propose a new two-stage framework (attention, then memory) that integrates these existing theories. Using a meta-analytic approach, we examine the extent to which the existing literature is able to test this two-stage framework. We show that including both attentional and memory factors explained variance in the interleaving effect better than attentional factors alone. However, as we also discovered, large gaps and confounded experimental designs in the category literature preclude strong tests of interactions that would be predicted from our new integrative two-stage model. We conclude with suggestions for future directions in the study of applied category learning.

\section{The Interleaving Effect}

We often think that to be a diligent, successful learner, we must focus, single-mindedly, on one thing at a time. In education, this assumption is manifest in different ways. For example, 
the concepts in our textbooks are blocked chapter by chapter, homework or practice worksheets often focus on one problem type at a time, and accelerated learning programs where students take just one short, intensive course at a time are gaining in popularity (e.g., intensive coding “bootcamps" like HackReactor, or Colorado College's "Block Plan" in which students only take one class at a time, five days per week for three and a half weeks). One of the more counterintuitive findings in the cognitive psychology literature, however, has been that interleaving study and practice of different concepts can benefit long-term learning ${ }^{1}$. The interleaving effect refers to the finding in the cognitive psychology literature that alternating between different concepts can benefit learning more than focusing on one concept at a time.

One important initial step is to establish whether a particular effect exists and to understand whether the effect is generalizable beyond the initially studied context. A recent meta-analysis of the interleaving effect (Brunmair \& Richter, 2019) found that there was a significant overall effect of interleaving (Hedges $g=.42$ ). They also found, however, significant heterogeneity of the effect size. Separating the existing literature by stimuli sets (paintings, naturalistic photos, artificial images, math-related tasks, expository texts, words, and tastes), the effect appeared reliable except for in the case of expository texts and words. As an instructional practice, interleaving promises huge potential for transforming learning. However, it is important to understand underlying mechanisms and boundary conditions before scaling to practice. Therefore, while documenting generalizability of an effect is important, an arguably more important goal is to be able to understand the processes that give rise to an effect and how they

\footnotetext{
${ }^{1}$ While recently popular in the educationally-relevant cognitive literature as it relates to concept and category learning, the idea that intermixing training confers benefits is not new. See contextual interference from early verbal learning research (Battig, 1966, 1978) and from motor skills training research (Lee, 1992; Shea \& Morgan, 1979) and catastrophic interference from connectionist network research (Maclelland, McNaughton \& O'Reilly, 1995; McCloskey \& Cohen, 1989).
} 
relate to the heterogeneity of an effect. Understanding the underlying mechanisms not only allow researchers to better predict the boundaries of generalizability, but they can also reveal more fundamental processes of human learning.

\section{An Emphasis on Underlying Processes}

While there are a surprising number of effects that generalize across both verbal and motor skills learning tasks, the extant literature has been careful to separate discussion of motor skills and verbal tasks (e.g., Soderstrom and Bjork, 2015; Seger \& Miller, 2010). Less care, however, has been taken with separating out the processes that underlie educationally-relevant cognitive (i.e., non-motor) tasks. Different educationally-relevant tasks, however, also involve different types of learning processes. For example, differentiating cancerous cells from noncancerous cells requires the ability to recognize and categorize different types of cells. A failure to correctly identify cells might be linked to a problem of discrimination and categorization. In contrast, being able to apply the appropriate mathematics formula to a particular mathematics problem requires that learners are not only able to recognize and categorize the type of problem that is presented, but also to be able to retrieve the correct procedure or formula for that type of problem, and to be able to correctly implement the procedural steps in the appropriate order. A failure to correctly solve a mathematics problem could be due to a problem of discrimination and categorization, but it could also be a problem with retrieval or implementation.

Brunmair and Richter (2019) chose not to include motor learning studies, correctly, in our view, as the evidence for dissociations between inductive conceptual learning and procedural learning is strong enough to warrant separate analyses. Nevertheless, Brunmair and Richter also included several tasks outside of the focus of our present review. In particular, their inclusion of tasks involving taste memory is not mirrored in the inclusion standards of the present meta- 
analysis, as taste memory most likely evolved to meet a set of specialized evolutionary constraints - for example maximizing enjoyment and nutrition, while maintaining aversion to toxins - distinct from the functions served and brain regions instantiated by other memorial processes (Lin, Arthurs, \& Reilly, 2017; Núñez-Jaramill et al., 2010; Palmerino, Rusiniak, \& Garcia, 1980). They also grouped stimuli by surface features rather than by underlying processes. For example, under mathematical tasks, they combined both studies in which category discrimination is emphasized (e.g., being able to recognize when different statistical tests are appropriate, Sana et al., 2017) with studies which also require procedural skills such as problem solving (Rohrer et al., 2014). Finally, they included some studies that were about memory rather than about category learning (e.g., Hausman \& Kornell, 2014), or in which blocked and interleaved schedules were not matched (e.g. studies that used adaptive algorithms, Rau et al., 2010).

\section{Attention-based Accounts of the Interleaving Effect}

In Brunmair and Richter (2019), the authors examined potential moderators of attentionbased accounts of the interleaving effect: namely, the discrimination hypothesis (that interleaving enhances attention to features that discriminate between different categories, Kang \& Pashler, 2012; Kornell \& Bjork, 2008) and its extension, the sequential attention hypothesis (that interleaving draws attention to between-category differences and that blocking draws attention to within-category similarities; Carvalho \& Goldstone, 2015, 2017). The discrimination hypothesis argues that the benefit of interleaving examples from different categories is that the juxtaposition of these different categories highlights the differences between them. Interleaving enhances discriminative contrasts, blocking does not. The sequential attention theory (Carvalho \& Goldstone, 2017) extends the discrimination hypothesis, positing that the sequence in which 
examples are studied influences the features on which a learner focuses their attention: Under interleaved sequences, where examples from different categories are juxtaposed, learners' attention is drawn to the features that help discriminate between the different categories (as in discriminative contrast theory). Under blocked sequences, where different examples from the same category are juxtaposed, learners' attention is drawn to the common features that define the category.

These attention-based theories hold implications for the types of moderators that would be important. Namely the moderators that have been identified include category similarity, temporal spacing, temporal juxtaposition and active (vs. passive) learning. Category similarity is thought to matter because the more similar the to-be-learned categories are to each other, the greater the interleaving benefit should be; in cases where the need for between-category discrimination is low or trivial, a blocking benefit might be obtained. Indeed this pattern of results is demonstrated in Carvalho and Goldstone (2014), who showed that high-similarity categories were better learned when interleaved while low-similarity categories (where withincategory commonalities were also more difficult to notice) were better learned when blocked. Temporal spacing (immediately sequential presentations versus spaced presentations) and temporal juxtaposition (simultaneous versus sequential presentations) are thought to matter because these manipulations either make it easier or more difficult for learners to attend to the important features (Birnbaum et al., 2013; Carvalho \& Goldstone, 2014; Kang \& Pashler, 2012; Sana et al., 2017; Zulkiply \& Burt, 2013): simultaneous presentations are more likely facilitate compare and contrast processes than sequential presentations, which in turn is more likely to facilitate these processes than spaced presentations. Finally, another corollary of the attentional bias hypothesis is that sequence effects should be larger under conditions of active learning than 
under conditions of passive learning, as under active learning, participants may be more likely to actively search for the key distinguishing features that discriminate between categories. On the other hand, during a passive learning task, participants might try to create a "positive characterization of each category" (Carvalho \& Goldstone, 2015, p. 282), and hence benefit from blocked sequencing.

\section{Memory-based Accounts of the Interleaving Effect}

Importantly however, there are also memory-based accounts of the interleaving effect that are missing from Brunmair and Richter's analyses. In fact, the last decade of interest in the interleaving effect arose as an extension of the spacing effect. The spacing effect (Carpenter, 2017; Cepeda et al., 2006) is one of the most robust effects in the cognitive psychology literature, and is the finding that distributing repetitions over time leads to better learning than massing those repetitions. Kornell and Bjork (2008) initially designed their experiments to be an illustration of a situation in which spacing learning out over time should not be beneficial for learning, hypothesizing that when category exemplars were spaced, it would make it difficult to identify commonalities and abstract the categorical information. Instead — to their surprise — the results revealed that spacing (or, what the literature now refers to as interleaving) category exemplars enhanced category induction.

Spacing is inherent to interleaved schedules: under interleaved schedules, examples from a given category are inherently distributed from each other. Spacing theories of interleaving extend the well-known benefits of spacing repetitions for long-term memory to the learning of categories, and predict that there should be an optimal level of spacing or difficulty (Cepeda et al., 2008). The study-phase retrieval hypothesis (Appleton-Knapp, Bjork, \& Wickens, 2005; Birnbaum, Kornell, Bjork, \& Bjork, 2013; Thios \& D’Agostino, 1976), for example, assumes 
that prior presentations are retrieved and elaborated on at the time of subsequent presentations (see also 'reminding theory' of spacing, Benjamin \& Ross, 2011). In other words, spacing out examples from a given category promotes retrieval or reminding of prior examples, which in turn strengthens learning; in contrast, blocking presentation of examples from the same category does not afford the forgetting that engages subsequent retrieval processes. Similarly, the retrieval effort hypothesis (Pyc \& Rawson, 2009) posits that the more effortful (but still successful) retrieval promoted by distributing examples leads to deeper processing. In massed or blocked sequences, the lack of spacing between presentations means that there is no need for retrieval or elaboration, and hence, no memorial benefit. When intervals are too long between examples from the same category, retrieval processes may fail, resulting in no additional learning benefit (Appleton-Knapp, Bjork, \& Wickens, 2005). What constitutes "too long," however, can be difficult to pinpoint. A related theory, the forgetting-as-abstraction hypothesis (Vlach, 2014), extends the study-phase retrieval theory for category learning: intervals between presentations of examples from the same category allow the learners to forget the category-irrelevant details that are specific to individual examples and to consolidate only the category-relevant details that are present across category examples (Vlach, 2014; Vlach \& Kalish, 2014).

These memory-based theories also hold implications for the types of moderators that would be important. Namely, that the effect may be moderated by the number of categories or the number of study examples and retention interval between end of study and final test. Spacing accounts predict that increasing the spacing between examples from a given category should improve learning. Birnbaum et al. (2013) tested this theory directly, by varying the average number of examples that intervened between two examples from the same category, while holding discrimination variability constant. They found that large spacing (15 intervening 
examples) led to better learning than did small spacing (three intervening examples). Most studies have not manipulated spacing in this way, but rather tend to interleave all categories together, which means that the number of categories can be used as a proxy for the spacing between stimuli from the same category. Memory-based theories would also imply that other important moderators include the number of presentations (i.e., examples or repetitions — the more repetitions, the larger the interleaving effect; Pavlik \& Anderson, 2005; Shaughnessy, Zimmerman \& Underwood, 1972; Underwood, 1970) and the retention interval (spacing effects should become stronger with at a delayed test compared to an immediate test; Cepeda et al, 2008; Rawson \& Kintsch, 2005).

\section{A Two-stage Framework of Sequencing Effects}

Although discrimination (i.e. attention-based) and spacing (i.e., memory-based) hypotheses have often been pitted against each other in the literature (e.g., Foster et al., 2019; Kang \& Pashler, 2012), the likely reality is that they are not mutually exclusive. We argue that the two types of processes are not competing, but rather sequential. That is, drawing from classical models of memory (e.g., multi-store model, Atkinson \& Shiffrin, 1968), it is likely that sequencing effects influence category learning in two stages: First, an attention-based stage of category discovery in which attention must be drawn to the relevant features. In this stage, sequencing effects are likely determined by category structure and similarity. Second, a memorybased stage in which learners need to memorize the cluster of features and the association between features and category labels: sometimes interleaving may be more beneficial; other times blocking may be more beneficial. In this stage, sequencing effects are likely to follow the same patterns as spaced learning effects in memory. Here, interleaving should be more beneficial. Finally, attention and memory processes are likely cyclical throughout the process of 
learning: The features that we attend to are more likely to be encoded into our memories, and our memories of past experiences likely shape how we attend to future stimuli (Kim \& Rehder, 2011).

Figure 1 is a schematic that illustrates some of the ways in which blocking or interleaving benefits may arise from this two-stage framework. What this schematic also illustrates is why the two-stage framework would make different predictions and situates different findings in the literature. In particular, the schematic illustrates the problem with pitting attention-based theories against memory-based theories. For example, the effect of inserting fillers in between to-bestudied category examples is often cited as evidence in favor of the attention-based theories (discriminative contrast or sequential attention theory) and as evidence against the memorybased theories. These studies show that if spacing disrupts juxtaposition and hence, contrastive processes, between examples from different categories (e.g., inserting trivia question fillers between study examples), then the interleaving benefit is eliminated (Birnbaum et al., 2013; Sana, Yan, \& Kim, 2017). Situated within the two-stage framework however, these results support the role of attention-based processes but do not constitute evidence against memorybased theories. Rather, if learning is gated at the attentional stage, then we would not expect spacing benefits to emerge at the subsequent memory stage either. In fact, in a different study, Birnbaum et al. (2013) found that when learning is not gated at the attentional stage (i.e., fillers were not inserted to disrupt discriminative contrasts between category examples), larger lags between examples of a given category leads to greater learning. This benefit cannot be attributed to an attentional process as in this study, as the number of discriminative contrasts afforded to the learner were the same between the short-lag condition and the long-lag condition; rather the benefit is attributed to memory processes. 
Conversely, if the to-be-learned categories are each distinct enough and directing learners' attention to the shared and discriminating features is unnecessary, then we might expect to find only benefits spaced (interleaved) practice on memory. Foster and colleagues (2019), for example, asked participants to practice four types of mathematics problems in an interleaved or blocked sequence. They chose a "critical" problem type and then varied whether the other three problem types were similar or dissimilar to the critical problem type. They found that the interleaving benefit was the same regardless of category set and argued that this supported the distributed-practice hypothesis over the discriminative contrast hypothesis. We interpret the results differently in our two-stage framework: For the similar set in which the categories were required discrmination, we propose that interleaving benefited learning at the attentional stage (as well as potentially the memory stage); for the dissimilar set in which categories were distinct, we propose that the attentional stage was unnecessary and hence, we see only benefits of spacing at the memory stage. That is, the same outcome- better performance following interleaved practice — could be attained via different pathways.

The implication of this two-stage framework is that researchers should pay more attention to the specific processes that might be required of any given type of learning content. For the purposes of conducting a meta-analysis of existing sequencing research, it also points to coding for memory-related experimental features and possible interactions. With a more complex model guiding predictions of sequencing effects, this raises another challenge for conducting a metaanalysis: It raises the need for a greater sampling of the experimental space. The goals of this present paper are therefore two-fold: (a) to examine both attention-based and memory-based moderators of the interleaving effect and (b) to identify the nature of existing studies and to the gaps that are currently missing in the literature. We begin by conducting a quantitative review. 


\section{Method}

As an exploratory step to understanding the simultaneous contributions of attentional and memory processes, we conducted a meta-analysis of the interleaving effect. We defined our parameters differently to those of Brunmair and Richter (2019) to focus more closely on the underlying processes and limiting our analysis only to studies that tested category learning. Most importantly, while Brunmair and Richter (2019) coded only moderators that tested attentionbased accounts, we also coded moderators that test memory-based accounts (see Tables S1 and S2 in Supplemental Online Materials for a summary of the moderators, the number of effect sizes, the sample size and the average effect sizes for each level of the moderators).

To keep the focus on our theoretical and methodological arguments about future directions for research, below, we support these arguments through the reporting of key metaanalytic findings, particularly in the form of reporting overall effects and multiple regression model comparison. Our method was pre-registered (https://osf.io/gsvu6/) and the full details of our meta-analysis method and the detailed results are reported in the Supplemental Online Materials. In other words, the main contribution of this review is not the specific outcome of the systematic quantitative review per se, but rather how the gaps can inform future research.

\section{Results}

\section{Overall Interleaving Effect Size}

A random effects model computed with the metafor $\mathrm{R}$ package (Viechtbauer, 2010; R Core Team, 2018) found that the overall effect size of the interleaving effect size, nesting effects $(\mathrm{k}=205)$ within publications $(k=61)$, was significantly larger than zero, $g=0.44,95 \% \mathrm{CI}=$

$[.34, .53], p<.001$. This effect size is very similar to that found in Brunmair and Richter (2019), 
$g=0.42$. It is important to remember that the overall effect size is computed with all studies, including those that would be predicted to find a benefit of blocking. Unsurprisingly, we also found a significant heterogeneity of effect sizes, $Q(204)=1213.03, p<.001$. From our univariate random effects model, we calculated $\mathrm{I}^{2}=86.70$, indicating 86.70 percent of the variance in outcomes was due heterogeneity (Viechtbauer, 2019). The forest plot is presented in Figure S1 in the Supplemental Online Materials, and the funnel plots are presented in Figure S2. We found no evidence of publication bias (see Supplemental Online Materials for full details).

\section{Multiple Regression Model Comparison}

There were substantial correlations between the different moderators that we coded in for our meta-analysis. The Cramer's $V$ correlations are reported in the correlation matrix depicted in Figure 2. Due to concerns with the confounded nature of moderators assessed in this metaanalysis, it would be inappropriate to rely on single moderator regression models, as these could be indicative of significant, yet spurious, relationships (Lipsey, 2003) ${ }^{2}$.

We built four nested mixed-effects regression models predicting the benefit of interleaving relative to blocking using the $R$ (R Core Team, 2018) package metafor (Viechtbauer, 2010). These models were: 1. Experimental (atheoretical); 2 Attention-based Factors; 3. Combined Memory-based and Attention-based Factors; and 4. A model incorporating the number of categories by similarity interaction term. Random effects were composed of experiments nested within paper. We report the relative fits of these models using likelihood tests, AIC, and BIC, when appropriate. These multiple regression analyses are informed by the

\footnotetext{
${ }^{2}$ Although separate tests of individual moderators may be significant, significance does not necessarily mean that the moderators, themselves, explain unique variance. Given the correlated nature of many of the moderators of interest, any single moderator model could actually reflect the contributions of multiple, correlated moderators.
} 
predominant theories of the effects of interleaving, however, they were not pre-registered, as we had not anticipated the high amount of confounding seen in the present dataset, which precluded single-moderator analysis. The results of each model and their fit indices are presented in Table 1.

\section{Attention and Memory Factors: Model Comparison}

Comparing the fit between Model 1 and Model 2 allows us to examine the contribution of the attention-based factors. Consistent with the findings from Brunmair and Richter (2019), incorporating attention-based factors (Model 2) led to a significantly better fit than incorporating only experimental factors (Model 1), $\chi^{2}=32.95, d f=4, p<.001$. Comparing the fit between Model 2 and Model 3 allows us to examine whether adding memory-related factors adds to variance explained, over and above the attentional factors. Overall, the best fitting model of the present meta-analytic data was Model 3, representing the combined contributions of memoryand attention-implicated moderators. Likelihood tests found that Model 3 fit better than Model 2, $\chi^{2}=21.00, d f=2, p<.001$. BIC and AIC also favored Model 3.

\section{Memory-Attention Interaction}

We pre-registered that we would explore two interactions. One was the interaction between the number of categories and supervised/unsupervised learning — however, there were not enough studies in which unsupervised learning was used. The other interaction we pre-registered was an interaction between the number of categories and similarity.

In our meta-analysis, we treat the number of categories as a proxy for spacing between examples from the same category. The two-stage framework would predict that benefits of increased number of categories (a process that benefits memory) would be most apparent for 
stimuli sets that are dissimilar, as those would not benefit from attention processes. We included this one interaction term in addition to the predictors of Model 3. We found that this interaction term was significant $(b=0.03, S E=.01, p=.017)$, indicating that the slope representing the benefit of the number of categories for the interleaving effect was steeper for dissimilar items ${ }^{3}$. This interaction is depicted in Figure 3. In other words, in support of dual processes of memory and attention, when the number of categories being studied increases, we see that the interleaving effect grows at a faster rate when the items are dissimilar. We did not explore any other potential interactions, however, due to limitations of the existing studies, which we describe in further detail in the next section.

\section{Discussion}

\section{Limitations of the Extant Empirical Literature}

Our quantitative review of the existing literature provided evidence that sequencing has a significant and multifaceted effect on category learning. On average, interleaving is beneficial, but that average is highly misleading. This basic finding is aligned with the findings of Brunmair and Richter (2019). What we additionally found — different from that of prior reviews - is that a model in which memory factors are added on top of attentional factors better accounts for the variance than attentional factors alone. In the midst of the present quantitative review, particularly as the result of analyzing our meta-analytic dataset, we realized that certain troubling patterns in the predominant manners of conducting interleaving experiments resulted in a far

\footnotetext{
${ }^{3}$ This model with the interaction term, however, was not significantly different from Model $3, \chi^{2}$ $=5.63, d f=2, p=.059$.
} 
lower level of generalizability of our findings than we had initially anticipated. We detail three core problems. First, a large proportion of the empirical literature uses the same or very similar experimental paradigms. Second, there is a serious problem of confounded moderators (Lipsey, 2003). Third, we find evidence of restricted ranges of moderators. That is, apart from the strong correlations between moderators of interest, many moderators have not been experimentally examined across their entire possible range of values. Each of these issues limits our ability to disambiguate the true effects of these moderators with implications for furthering theoretical understanding and practical generalization. In the following sections, we detail each of these issues.

\section{Proliferation of a Single Paradigm}

One particular paradigm represented a large proportion of the effect sizes. Specifically, 36 out of 100 of the published effect sizes and 89 out of 209 of the total (published and unpublished) effect sizes used paintings stimuli and passive study. Moreover, when painting stimuli were used, the number of categories was usually $12(k=50)$. In the interest of full disclosure, many of these were associated with the first author of this present paper $(k=37)$. In other words, experimental design seems to be biased in the distribution of underlying experimental variable values. Similar to how many participants are sampled from a convenient source (college psychology courses), there is a tendency towards convenience sampling of experimental designs. We do not believe that this tendency is unique to a particular researcher, nor to the interleaving literature; rather this is likely a typical feature of most empirical psychology research. Indeed, it is arguably a rigorous way of proceeding with research: When an effect is first described using a particular paradigm, it is important to replicate it as closely as possible (Pashler \& Harris, 2012). Retaining the same paradigm is also then important for testing 
underlying processes: researchers can systematically change one experimental feature at a time, testing whether that change yields differences in the experimental outcome. If research were not paradigmatic in this way, it would be difficult to isolate why an outcome has been amplified or attenuated. Yet, such a paradigmatic focus becomes problematic for generalization and situating effects in a broader understanding of learning processes. Moreover, as we found in our review, researchers rarely explore the experimental design space in a systematic fashion.

\section{Confounded Moderators Leave Gaps in the Evidence Base}

One of the consequences of having a bias in experimental design is that potentially important moderators may become confounded within the literature space. Although the twostage framework makes predictions about interactions, we were limited in our ability to explore these in our quantitative review as a result of confounded moderators and non-systematic manipulation of important variables (e.g., number of categories, active or passive participation).

Confounded moderators limit the conclusions that can be drawn broadly concerning the relative importance of likely explanatory factors. Figure 2 presents a Cramer's $V$ pairwise correlation matrix between our moderators. Cramer's $V$ is a non-parametric measure of association based upon the chi-squared statistic, which measures the association between nominal variables and always results in a value between 0 and 1 (there are no negative Cramer's $V$ statistics; hence it is non-directional). Two advantages of using Cramer's $V$ measure is that it is better at dealing with categorical variables (as most of our moderators are) and does not assume linear relationships. Generally, $V$ values between 0.1 and 0.3 are considered small effect sizes, between 0.3 and 0.5 considered medium, and greater than 0.5 considered large (Osteen \& Bright, 2010; Kotrlik, Williams, \& Jabor, 2011). As this matrix shows, the associations between these variables and others are extremely high, precluding clear interpretation in several cases. Of the 
66 correlations reported in Figure 2, $42 \%$ were of medium size ( $V$ s between 0.3 and 0.5 ) and $15 \%$ were large ( $V$ s greater than or equal to 0.5$)$. This high correlation between moderators is a known problem in meta-analysis (e.g., Lipsey, 2003), and we implemented one of the proposed solutions (multivariate analysis). However, the broader problem is that the data available to go into the meta-analysis is consistently confounded.

In Figure 4, we provide four examples that further illustrate the issue with confounded moderators. Each panel in Figure 4 displays a combination of three moderators, with each point in the plot representing an effect size within our meta-analysis dataset. These four panels are not a randomly selected set of moderators; rather, we selected combinations of moderators that are relevant to the two-stage framework. Panel A plots a key moderator within attention-based hypotheses (similarity of the categories) against a key moderator within the memory-based hypotheses (number of categories), by stimuli type (visual or cognitive) - this represents the interaction that we tested in our meta-analysis. Although we did find that this interaction was significant, what is immediately apparent from Panel A is that (a) very few studies have used more than 8 or 12 categories; and (b) experiments using cognitive stimuli are particularly illrepresented across the range of number of categories - rather, most of the studies using cognitive stimuli present participants with only between 2-4 categories.

The gaps in the evidence base are more apparent in the other three panels: Panel B reveals that no interleaving studies using cognitive stimuli has presented examples simultaneously, even though there is a large body of research on category learning on simultaneous versus sequential study (e.g., Birnbaum et al., 2013; Carvalho \& Goldstone, 2014; Kang \& Pashler, 2012). Panels C and D of Figure 4 both point to the insufficiency of existing studies to test certain predicted interactions of the two-stage framework: the interaction between 
category similarity and presence of interfiller trials, and the interaction between category similarity and presentation simultaneity. If to-be-learned categories are similar and difficult to discriminate, then learners' attention to the critical distinguishing features will be hindered if an intertrial filler is inserted. Hence, we would expect an intertrial filler to attenuate the interleaving effect when categories are similar. On the other hand, if the to-be-learned categories are dissimilar, then we would expect intertrial fillers to amplify the interleaving effect via memory processes. Similarly, when categories are similar, category learning will benefit primarily from attentional processes. Interleaving under both simultaneous and sequential presentation allows learners to attend to the features that distinguish categories, and hence an interleaving benefit should emerge in both presentation modes. When categories are dissimilar, the need for attentional processes is low and hence, category learning will benefit primarily from memory processes. Interleaving under simultaneous presentation is much less likely to engage retrieval or memory processes and hence, an interleaving benefit should emerge only under sequential presentation. As Panels C and D show, however, there were almost no studies in which experimenters tested the effect of sequencing on the learning of dissimilar categories with intertrial fillers and there was not a single study in which categories were dissimilar and simultaneously presented.

\section{Issues of Restricted Range}

During the course of this research, it became apparent that the extant range of values for important moderators is restricted. We will focus on the number of categories as an example moderator. However, this issue of restricted range is also relevant to other moderators, including those that are typically treated categorically (e.g., similarity) that should perhaps be placed within a continuous range of similarity. Moderator values were also frequently set to one of a 
few values (e.g., 6 or 12 for the number of categories). Restricting the range of values can be convenient for executing standard experimental designs (e.g., making the number of categories divisible by 4 to accommodate a $2 \times 2$ design), but also carry consequences. Restricting the range of values for a moderator impedes theoretical development and practical application. In essence, restricting the range of potentially important moderators restricts the range of potentially important conclusions as well. This is not to say that moderators must be manipulated in all experiments, but rather that if very few researchers (or none) manipulate a moderator, the possibility of generalization is significantly curtailed. For instance, regarding meta-analysis and theory-building, failing to explore the effects of greater or fewer categories across or within experiments makes it challenging to know when and how attentional mechanisms that may underlie interleaving effects actually operate. Participants may compare across exemplars, but are these benefits influenced by the variety and number of recent examples from which to compare? To what extent and under what conditions does a participants' own individual abilities (e.g., operation span, Kane \& Engle, 2003) become relevant? These are important questions that need very specific answers in order to broadly benefit education. In short, restricting the range of values contributes to impeding theoretical development that would help educators and developers of educational technology understand if, and, the extent to which students should interleave.

\section{The Two-Stage Framework Roadmap for Future Research}

The two-stage framework is naturally cyclical; attention and memory interact dynamically over time (Turke-Brown, 2007). This framework is in progress, and future research is needed to fill in blank spots in the relations between attention and memory to elucidate how best to schedule practice for students to learn categories. Our meta-analysis confirmed some 
findings, but also revealed shortcomings in experimental design. Specifically, attention and memory are frequently studied separately (attention more so than memory), but should be studied together in order to give a more complete picture of category learning. Below we describe several possibilities for future research that could account for the issues described above (proliferation of a single paradigm, confounded moderators, restricted range) and understand interleaving as inherently involving both attention and memory factors.

\section{Broadening the Stimulus Space}

One of the issues we identified was a proliferation of a single experimental paradigm - a substantial amount of interleaving research on category learning has used a small number of stimuli, especially paintings, birds, and butterflies. This is likely attributable to convenience and a desire to relate findings to prior work (e.g., that also used paintings). However, if the goal is to generate prescriptions for how to best teach categories (even visual categories), constraining research to a small number of stimuli is likely to introduce idiosyncratic stimuli-specific biases in experimental findings. Such constraints also may limit the ability of researchers to properly manipulate important variables (e.g., similarity, number of categories). Even if for example the similarity among paintings was quantified, and a large number of painters (and paintings) were included, generalization would be difficult. In short, a broad swath of stimuli need to be utilized to evaluate whether broad claims can be made about how to implement interleaving.

\section{Parametric Manipulations of Critical Variables}

In addition to broadening the stimulus space, it may also be important to systematically manipulate levels of the existing variables. Under parametric manipulation, variables of interest are systematically tested across multiple levels of intensity (Brand et al., 2019), in opposition to 
the two-armed A/B experimental design traditionally employed in cognitive psychology. In interleaving studies, this might look like implementing experimental designs where the rate of interleaving is manipulated across a range of values, or likewise, the number of categories. Critically, this will allow testing of potentially important interactions between variables. Parametric manipulation has been used to great effect in neighboring research areas of cognitive psychological research, most notably in Cepeda et al.'s (2008) investigation of the spacing effect in which the researchers systematically varied multiple levels of spacing intervals with multiple levels of retention interval (yielding 26 different conditions).

We recommend conducting experiments with parametric manipulations, because this approach would allow for greater exploration of the experiment-space that may be relevant for understanding the effect of interleaving. The modal interleaving study in our review used paintings as stimuli, had six categories per condition, and treated interleaving as a binary factor (interleaved or blocked). However, extant theories aiming to explain interleaving imply that all of these attributes (stimuli, number of categories being learned, rate of interleaving) may interact. For example, if attention across exemplars facilitates comparison and possibly category learning when exemplars are interleaved, the number of categories will naturally influence the efficacy of comparing exemplars. Relatedly, working memory capacity may be differentially relevant depending on the similarity and number of categories. Thus, the similarity, number of categories, and rate of interleaving need to be considered simultaneously. Successful implementation may entail collaborative multi-site projects with large samples sizes (e.g., Klein et al., 2014) and alternative experimental designs. 


\section{Alternative Experimental Designs}

The non-systemic traversal of the interleaving experiment-space ${ }^{4}$ is likely to be suboptimal for determining when and the extent to which interleaving should be applied. Given existing experimental preferences (e.g., choosing a particular rate of interleaving and number of categories and comparing to a blocked condition), a complete understanding of interleavingeven with the use of parametric experimental manipulations - could require gigantic sample sizes and decades (or longer) of careful research. However, if the goal is to understand the functional relationship between, for example, the number of categories and (rate of) interleaving, there may be more efficient methods for experimental design. For example, Lindsay, Mozer, Huggins, and Pashler (2013) sought to optimize the balance between the rate of interleaving and amount of fading (e.g., starting with easy examples and increasing difficulty). Their aim was to devise a method to avoid running large A/B style tests comparing different values of each variable against each other, and instead assume there was a functional relationship that could be traversed to find an optimum. For example, instead of manipulating just two levels of each variable (with a large number of participants at each level), multiple levels of each variable are chosen (with a smaller number of participants at each level) to allow researchers to model learning. Such a method might preclude pairwise comparison of points on that functional line depending on statistical power, but overall would give stronger evidence about the relationship among the variables of interest. Other recent research has shown how adaptive experimental design can be more efficient when using multi-armed bandit algorithms (Rafferty, Ying, \& Williams, 2019). Adopting alternative approaches may help avoid some of the pitfalls that have

\footnotetext{
${ }^{4}$ An example coordinate in this experiment-space could be high similarity among categories, ten to-be-learnedcategories, and an intermediate rate of interleaving (e.g., alternate categories $50 \%$ of the time), etc.
} 
become common in many areas of social science (Watts, 2017) and get to the heart of answering the question "how and when should interleaving be applied?".

Testing Memory Factors. While our first three recommendations are those that apply more broadly to the field, our review has also indicated several ways forward for testing theory specific to the interleaving effect. Our quantitative analysis indicated that more categories predicted a larger effect of interleaving. This finding supports a spacing effect (more categories being interleaved leads to more spacing between repetitions of the same category), and by extension, the contribution of memory processes. But under what circumstances would more categories leading to a larger interleaving effect be explained by attentional theories? Typically, such theories concern comparisons a participant could make between the current exemplar they are viewing and the immediately preceding exemplar (within or between categories depending on rate of interleaving). In fact, there is evidence that disrupting comparison with the immediately preceding exemplar (by inserting fillers) can eliminate the benefit of interleaving in some cases (Birnbaum, 2013). However, if attentional explanations are mostly concerned with attention towards immediately preceding examples, it is not clear how a purely attentional explanation of interleaving could explain why increasing the number of categories (e.g., from 6 to 12) increases the benefit of interleaving. Of course, a participant may be reminded of prior (nonadjacent) exemplars, and compare them to the present exemplar under consideration. That process would involve recall, and thus spacing would become an additional relevant factor. However, to our knowledge, there are no experiments directly testing how spacing and attentional mechanisms may together contribute to interleaving effects. For instance, an experiment could insert inter-trial fillers while manipulating the number of categories and retention interval. Most interleaving experiments seem to have been tailored for studying 
attentional rather than memory effects (e.g., $90 \%$ of the experiments in our meta-analysis have a retention interval of less than 24 hours).

Hybrid and Adaptive Schedules. A particularly relevant example connecting attention and memory is how expertise influences information-processing. Expertise is known to be highly relevant for both how attention is devoted and what is retained. This is clear in educational research - expertise can change what instructional format is most beneficial for learning (Cronbach \& Snow, 1977; Kalyuga, Ayres, Chandler \& Sweller, 2003). Experts generally process information relevant to their domain differently, such as preferentially directing attention to more relevant features (Kim \& Rehder, 2011) and chunking information more effectively (Chase \& Simon, 1973). This interaction between expertise (prior learning), attentional allocation, and subsequent learning is likely to be highly relevant for determining optimal interleaving schedules. As knowledge is acquired, participants' attention will be directed differently and thus scheduling that most prior research has shown to be effective for naive learners may become inefficient. For example, blocking may be initially preferable due to dissimilar category structure, but once the participant learns relevant category features enabling across category comparison, interleaving may be introduced to future improve learning via spacing and across-category comparisons. Future educational research should explore the efficacy of this approach by manipulating category similarity as well as altering the schedule according to student performance. More precise adaptive scheduling could be achieved with a computational model. However, although there are recent models that account for category similarity (Carvalho \& Goldstone, 2019) and others that track memory as a function of spacing (Walsh et al, 2018; Pavlik, Eglington, \& Harrell-Williams, 2020; Eglington \& Pavlik, 2020), to our knowledge there are no models that explicitly account for both simultaneously. 


\section{Conclusion}

For the present review, we initially set out to meta-analyze the interleaving literature. We found a medium beneficial effect of interleaving, moderated by several important factors. Our analysis also revealed that multiple processes may contribute to explaining the interleaving effect. In contrast to prior work, we coded for moderators that are likely to be relevant for both memory-based accounts (e.g., number of categories) and those more relevant to attention-based accounts (e.g., similarity, inter-trial fillers). In line with prior research, our analyses indicated that interleaving enabled attentional mechanisms that promoted learning, as long as the categories themselves had the proper similarity structure. However, interleaving also naturally increases spacing, and, to add to existing literature, we found evidence that this spacing also contributes to the interleaving effect. In other words, we found evidence that both attentional and spacing accounts are partially supported, which should not be entirely surprising, given the interplay between attention and memory.

However, coding this literature also revealed significant issues concerning how interleaving research is carried out, especially the importance of more systematic and comprehensive manipulation of relevant variables and alternative experimental designs to further elucidate how to effectively implement interleaved practice. For example, despite the known relationship between the similarity structure of learning materials and the effect of interleaving (Carvalho \& Goldstone, 2014), the similarity among categories has rarely been quantified (or manipulated directly), and a significant plurality of research has been with specific sets of stimuli. Our two-stage framework lays out a roadmap for possible interactions to explore, but these were interactions that we could not examine in the present review given gaps in the existing evidence base. The non-systematic nature of experimental design within the interleaving 
literature hence makes it more challenging to broadly interpret overall findings and develop specific theories and models that can guide future research and pedagogy. On a more positive note, these issues highlight new areas of research and motivate new experiments that may help further refine the two-stage framework (depicted in Figure 1), and lead to improved pedagogical decision-making for how to best learn categories. 


\section{References}

Abushanab, B., \& Bishara, A. J. (2013). Memory and metacognition for piano melodies: Illusory advantages of fixed- over random-order practice. Memory \& Cognition, 41(6), 928-937. https://doi.org/10.3758/s13421-013-0311-z

Ashby, F. G., Alfonso-reese, L. A., Turken, U., \& Waldron, E. M. (1998). A neuropsychological theory of multiple systems in category learning. Psychological Review, 442-481.

Ashby, F. G., \& Maddox, W. T. (2005). Human Category Learning. Annual Review of Psychology, 56(1), 149-178. https://doi.org/10.1146/annurev.psych.56.091103.070217

Birnbaum, M. S., Kornell, N., Bjork, E. L., \& Bjork, R. A. (2013). Why interleaving enhances inductive learning: The roles of discrimination and retrieval. Memory \& Cognition, 41(3), 392-402. https://doi.org/10.3758/s13421-012-0272-7

Carvalho, P. F., \& Goldstone, R. L. (2014). Putting category learning in order: Category structure and temporal arrangement affect the benefit of interleaved over blocked study. Memory \& Cognition, 42(3), 481-495. https://doi.org/10.3758/s13421-013-0371-0

Carvalho, P. F., \& Goldstone, R. L. (2015). The benefits of interleaved and blocked study: Different tasks benefit from different schedules of study. Psychonomic Bulletin \& Review, 22(1), 281-288. https://doi.org/10.3758/s13423-014-0676-4

Carvalho, P. F., \& Goldstone, R. L. (2017). The sequence of study changes what information is attended to, encoded, and remembered during category learning. Journal of Experimental Psychology: Learning, Memory, and Cognition, 43(11), 1699-1719. https://doi.org/10.1037/xlm0000406

Carvalho, P. F., \& Goldstone, R. (2019, September 13). A computational model of contextdependent encodings during category learning. https://doi.org/10.31234/osf.io/8psa4 
Cepeda, N. J., Vul, E., Rohrer, D., Wixted, J. T., \& Pashler, H. (2008). Spacing Effects in Learning: A Temporal Ridgeline of Optimal Retention. Psychological Science, 19(11), 1095-1102. https://doi.org/10.1111/j.1467-9280.2008.02209.x

Chase, W. G., \& Simon, H. A. (1973). Perception in chess. Cognitive psychology, 4(1), 55-81.

Chun, M. M., \& Turk-Browne, N. B. (2007). Interactions between attention and memory. Current opinion in neurobiology, 17(2), 177-184.

Cronbach, L., \& Snow, R. (1977). Aptitudes and instructional methods: A handbook for research on interactions. New York: Irvington.

Dunlosky, J., Rawson, K. A., Marsh, E. J., Nathan, M. J., \& Willingham, D. T. (2013). Improving Students’ Learning With Effective Learning Techniques: Promising Directions From Cognitive and Educational Psychology. Psychological Science in the Public Interest, 14(1), 4-58. https://doi.org/10.1177/1529100612453266

Eglington, L. G., \& Kang, S. H. K. (2017). Interleaved Presentation Benefits Science Category Learning. Journal of Applied Research in Memory and Cognition, 6(4), 475-485. https://doi.org/10.1016/j.jarmac.2017.07.005

Eglington, L. G., \& Pavlik Jr, P. I. (2020). Optimizing practice scheduling requires quantitative tracking of individual item performance. npj Science of Learning, 5(1), 1-10. https://doi.org/10.1038/s41539-020-00074-4

Hall, K. G., Domingues, D. A., \& Cavazos, R. (1994). Contextual interference with skilled baseball players. Perceptual and Motor Skills, 78, 835-841.

Jolly, E., \& Chang, L. J. (2019). The Flatland Fallacy: Moving Beyond Low-Dimensional Thinking. Topics in Cognitive Science, 11(2), 433-454.

Kalyuga, S., Ayres, P., Chandler, P., \& Sweller, J. (2003). The expertise reversal effect. 
Educational Psychologist, 38(2), 3-31.

Kane, M. J., \& Engle, R. W. (2003). Working-memory capacity and the control of attention: the contributions of goal neglect, response competition, and task set to Stroop interference. Journal of experimental psychology: General, 132(1), 47-70. https://doi.org/10.1037/0096-3445.132.1.47

Kang, S. H. K., \& Pashler, H. (2012). Learning painting styles: Spacing is advantageous when it promotes discriminative contrast. Applied Cognitive Psychology, 26(1), 97-103. https://doi.org/10.1002/acp.1801

Kim, S., \& Rehder, B. (2011). How prior knowledge affects selective attention during category learning: An eyetracking study. Memory \& Cognition, 39(4), 649-665.

Klein, R. A., Ratliff, K. A., Vianello, M., Adams, R. B., Jr., Bahník, Š., Bernstein, M. J., . . . Nosek, B. A. (2014). Investigating variation in replicability: A “many labs" replication project. Social Psychology, 45(3), 142-152. http://dx.doi.org/10.1027/1864-9335/a000178

Kornell, N., \& Bjork, R. A. (2008). Learning Concepts and Categories: Is Spacing the "Enemy of Induction"? Psychological Science, 19(6), 585-592. https://doi.org/10.1111/j.14679280.2008.02127.x

Kotrlik, J. W., Williams, H. A., \& Jabor, M. K. (2011). Reporting and Interpreting Effect Size in Quantitative Agricultural Education Research. Journal of Agricultural Education, 52(1), 132-142.

Mayfield, K. H., \& Chase, P. N. (2002). The effects of cumulative practice on mathematics problem solving. Journal of Applied Behavior Analysis, 35(2), 105-123. https://doi.org/10.1901/jaba.2002.35-105

Lindsey, R. V., Mozer, M. C., Huggins, W. J., \& Pashler, H. (2013). Optimizing instructional 
policies. In Advances in neural information processing systems (pp. 2778-2786).

McCabe, J. (2011). Metacognitive awareness of learning strategies in undergraduates. Memory \& Cognition, 39(3), 462-476. https://doi.org/10.3758/s13421-010-0035-2

Noh, S. M., Yan, V. X., Bjork, R. A., \& Maddox, W. T. (2016). Optimal sequencing during category learning: Testing a dual-learning systems perspective. Cognition, 155, 23-29. https://doi.org/10.1016/j.cognition.2016.06.007

Ollis, S., Button, C., \& Fairweather, M. (2005). The influence of professional expertise and task complexity upon the potency of the contextual interference effect. Acta Psychologica, 118(3), 229-244. https://doi.org/10.1016/j.actpsy.2004.08.003

Osteen, P., \& Bright, C. Effect Sizes and Intervention Research. Society for Social Work and Research Conference. San Francisco, CA. January 14-17, 2010. https://archive.hshsl.umaryland.edu/handle/10713/3582

Ostrow, K., Heffernan, N., Heffernan, C., \& Peterson, Z. (2015). Blocking Vs. Interleaving: Examining Single-Session Effects Within Middle School Math Homework. In C. Conati, N. Heffernan, A. Mitrovic, \& M. F. Verdejo (Eds.), Artificial Intelligence in Education (pp. 338-347). Springer International Publishing.

Pashler, H., \& Harris, C. R. (2012). Is the replicability crisis overblown? Three arguments examined. Perspectives on Psychological Science, 7(6), 531-536.

Pavlik Jr, P. I., Eglington, L. G., \& Harrell-Williams, L. M. (2020, May 2). Generalized Knowledge Tracing: A Constrained Framework for Learner Modeling. arXiv preprint arXiv:2005.00869.

Rafferty, A., Ying, H., \& Williams, J. (2019). Statistical Consequences of using Multi-armed Bandits to Conduct Adaptive Educational Experiments. JEDM $\mid$ Journal of Educational 
Data Mining, 11(1), 47-79. https://doi.org/10.5281/zenodo.3554749

Rau, M. A., Aleven, V., \& Rummel, N. (2013). Interleaved practice in multi-dimensional learning tasks: Which dimension should we interleave? Learning and Instruction, 23, 98114. https://doi.org/10.1016/j.learninstruc.2012.07.003

R Core Team (2018). R: A Language and Environment for Statistical Computing. R Foundation for Statistical Computing, Vienna, Austria. https://www.R-project.org/.

Rohrer, D., Dedrick, R. F., Hartwig, M. K., \& Cheung, C.-N. (2019). A randomized controlled trial of interleaved mathematics practice. Journal of Educational Psychology. https://doi.org/10.1037/edu0000367

Rohrer, D., \& Taylor, K. (2007). The shuffling of mathematics problems improves learning. Instructional Science, 35(6), 481-498. https://doi.org/10.1007/s11251-007-9015-8

Rohatgi, A. (2018). WebPlotDigitizer - Web Based Plot Digitizer. Austin, Texas, USA. Retrieved from https: //automeris.io/WebPlotDigitizer

Sana, F., Yan, V. X., \& Kim, J. A. (2017). Study sequence matters for the inductive learning of cognitive concepts. Journal of Educational Psychology, 109(1), 84-98. https://doi.org/10.1037/edu0000119

Sana, F., Yan, V. X., Kim, J. A., Bjork, E. L., \& Bjork, R. A. (2018). Does Working Memory Capacity Moderate the Interleaving Benefit? Journal of Applied Research in Memory and Cognition, 7(3), 361-369. https://doi.org/10.1016/j.jarmac.2018.05.005

Tauber, S. K., Dunlosky, J., Rawson, K. A., Wahlheim, C. N., \& Jacoby, L. L. (2013). Selfregulated learning of a natural category: Do people interleave or block exemplars during study? Psychonomic Bulletin \& Review, 20(2), 356-363. https://doi.org/10.3758/s13423012-0319-6 
Vlach, H. A. (2014). The Spacing Effect in Children's Generalization of Knowledge: Allowing Children Time to Forget Promotes Their Ability to Learn. Child Development Perspectives, 8(3), 163-168. https://doi.org/10.1111/cdep.12079

Watts, D. J. (2017). Should social science be more solution-oriented?. Nature Human Behaviour, l(1), 0015 .

Wolfe, J. M. (2019). Visual Attention: The Multiple Ways in which History Shapes Selection. Current Biology, 29(5), R155-R156.

Yan, V. X., Bjork, E. L., \& Bjork, R. A. (2016). On the difficulty of mending metacognitive illusions: A priori theories, fluency effects, and misattributions of the interleaving benefit. Journal of Experimental Psychology: General, 145(7), 918-933. https://doi.org/10.1037/xge0000177

Yan, V. X., Soderstrom, N. C., Seneviratna, G. S., Bjork, E. L., \& Bjork, R. A. (2017). How should exemplars be sequenced in inductive learning? Empirical evidence versus learners' opinions. Journal of Experimental Psychology: Applied, 23(4), 403-416. https://doi.org/10.1037/xap0000139

Viechtbauer, W. (2010). Conducting meta-analyses in R with the metafor package. Journal of Statistical Software, 36(3), 1-48. URL: http://www.jstatsoft.org/v36/i03/

Viechtbauer, W. (2019). $I^{\wedge} 2$ for Multilevel and Multivariate Models. The Metafor package: A meta-analysis package for $R$. http://www.metaforproject.org/doku.php/tips:i2_multilevel_multivariate

Zulkiply, N., \& Burt, J. S. (2013). The exemplar interleaving effect in inductive learning: Moderation by the difficulty of category discriminations. Memory \& Cognition, 41(1), 16-27. https://doi.org/10.3758/s13421-012-0238-9 
Zulkiply, N., McLean, J., Burt, J. S., \& Bath, D. (2012). Spacing and induction: Application to exemplars presented as auditory and visual text. Learning and Instruction, 22(3), 215221. https://doi.org/10.1016/j.learninstruc.2011.11.002 
Table 1

Comparison of Multiple Regression Results

\begin{tabular}{|c|c|c|c|c|}
\hline Predictor $($ Value = 1) & 0. Null Model & $\begin{array}{l}\text { 1. Expt. } \\
\text { Factors }\end{array}$ & $\begin{array}{l}\text { 2. Attentional } \\
\text { Factors }\end{array}$ & $\begin{array}{l}\text { 3. Attentional and } \\
\text { Memory Factors }\end{array}$ \\
\hline Intercept & $0.45(.05) * * *$ & $0.81(0.16) * * *$ & $0.83(0.17) * * *$ & $0.26(.13) *$ \\
\hline Schedule Manipulation (Within) & & $-0.12(0.09)$ & $-0.11(0.09)$ & $-0.14(.08)$ \\
\hline Publication Status (Published) & & $-0.10(.09)$ & $-0.09(0.10)$ & $-0.07(.09)$ \\
\hline Stimuli Type (Cognitive) & & $-0.45(0.12) * * *$ & $-0.49(0.12) * * *$ & $-0.25(.12) *$ \\
\hline Population Pool (MTurk) & & $-0.26(0.09) * *$ & $-0.28(0.10) * *$ & $-0.18(.09) *$ \\
\hline \multicolumn{5}{|l|}{ Sequential or Simultaneous } \\
\hline Presentation (Simultaneous) & & & $0.07(0.09)$ & $0.09(.09)$ \\
\hline Intertrial Filler (Yes) & & & $-0.26(0.12) *$ & $-0.26(0.11) *$ \\
\hline Similarity (Dissimilar) & & & $\mathbf{- 0 . 3 2}(.06) * * *$ & $-0.33(0.06) * * *$ \\
\hline Study Type (Active) & & & $0.07(.08)$ & $0.10(0.08)$ \\
\hline Retention Interval ( $>=1$ Day) & & & & $0.01(0.10)$ \\
\hline Number of Categories & & & & $0.04(0.01) * * *$ \\
\hline \multicolumn{5}{|l|}{ Model Fit Statistics } \\
\hline Negative Log Likelihood & 193.40 & 183.80 & 168.47 & 157.63 \\
\hline AIC & 392.80 & 381.61 & 358.93 & 341.25 \\
\hline $\mathrm{BIC}$ & 402.68 & 404.66 & 395.16 & 384.06 \\
\hline
\end{tabular}

Note. Model fit statistics, such as negative log likelihood, AIC, and BIC, can be used to compare the relative fits of each model, with lower scores indicating better fits.

$* p<.05, * * p<.01, * * * p<.001$. 


\section{Figure 1}

Schematic of the Two-stage Framework of Sequencing Effects

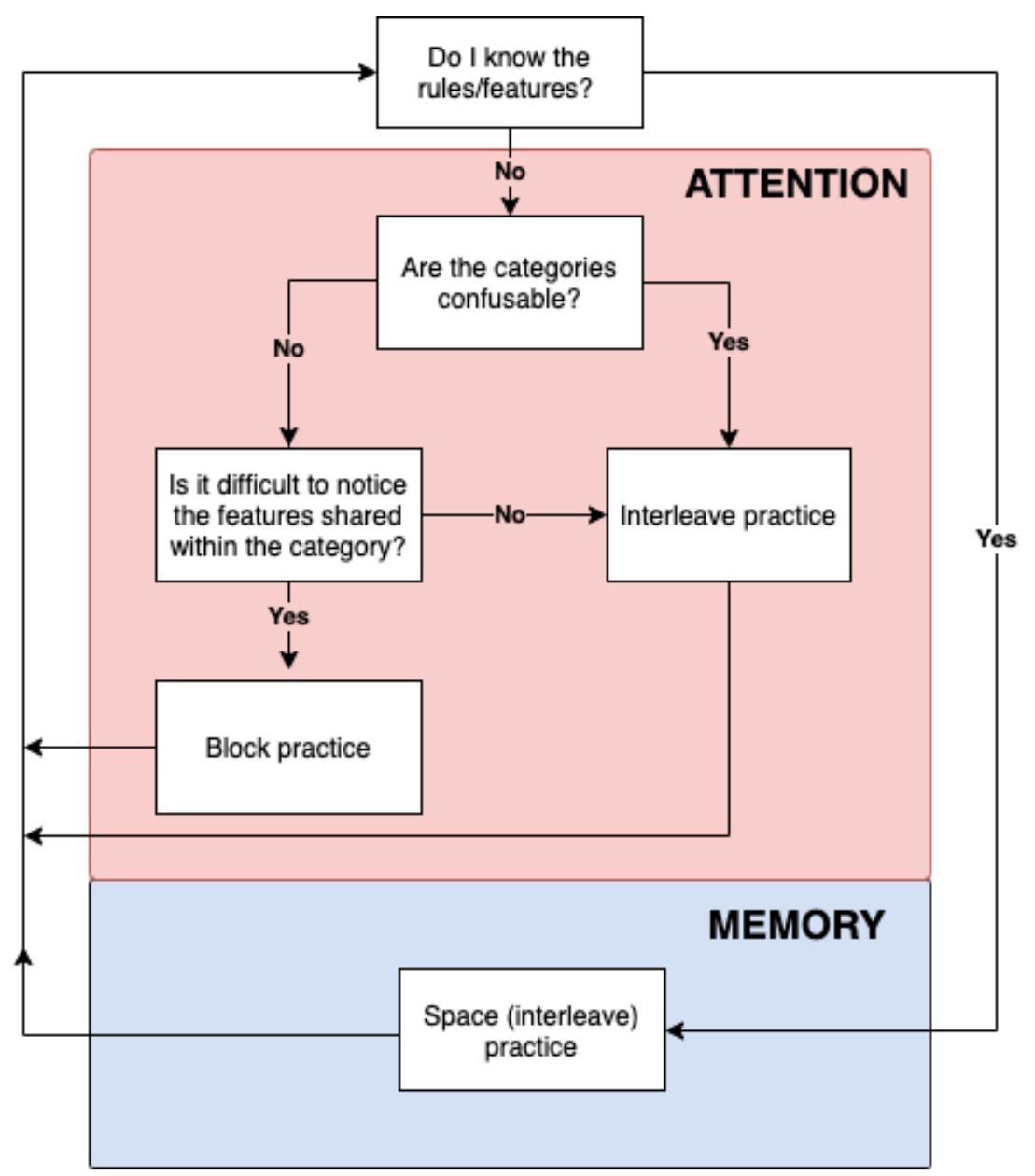




\section{Figure 2}

Cramer's V Correlation Matrix between Moderators

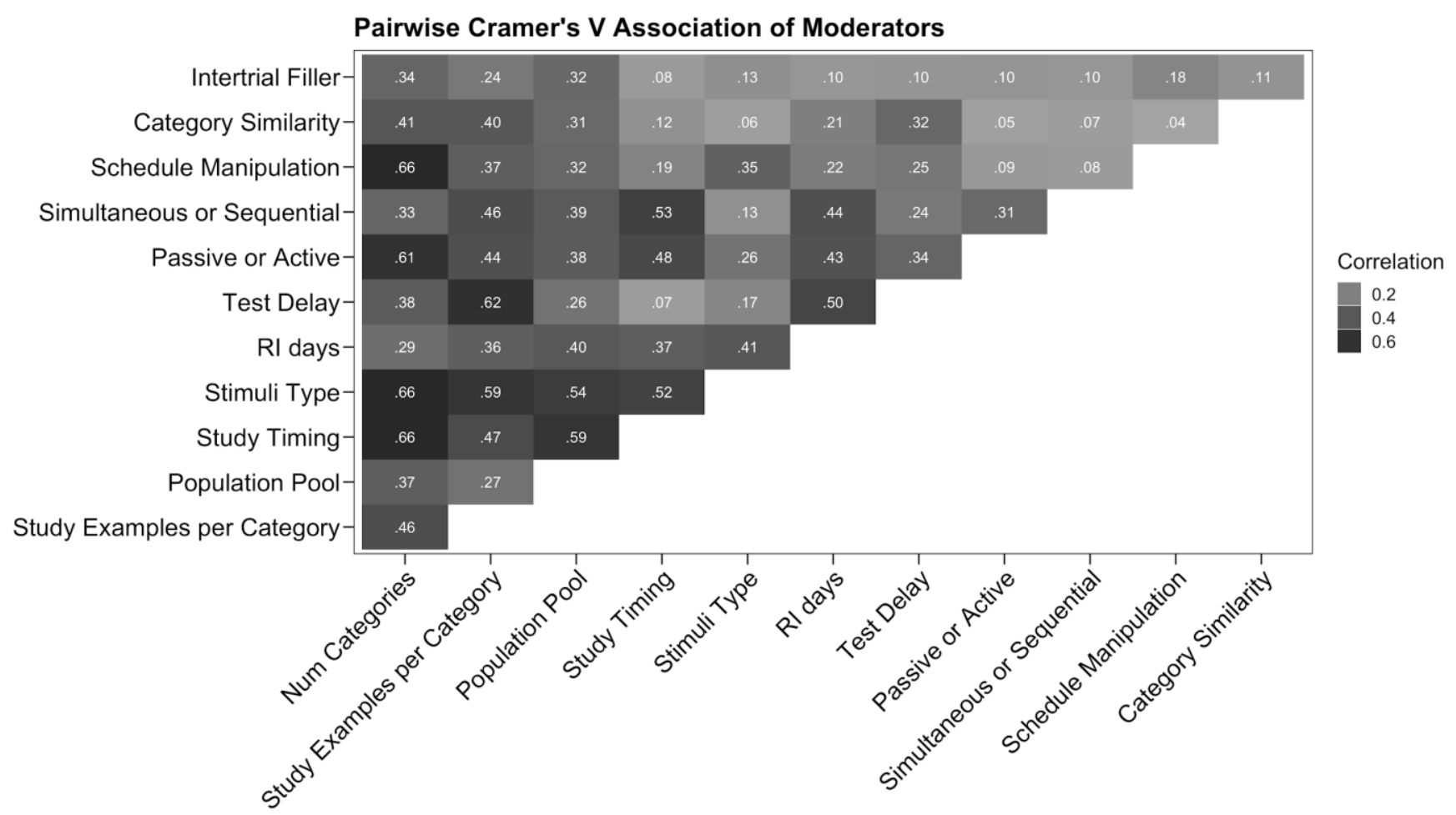

Note. Cramer's $V$ is a non-parametric correlation statistic. The columns representing each moderator are ordered from left-to-right, most-to-least correlated. 


\section{Figure 3}

Interaction Between Number of Categories and Similarity on Interleaving Effect Size

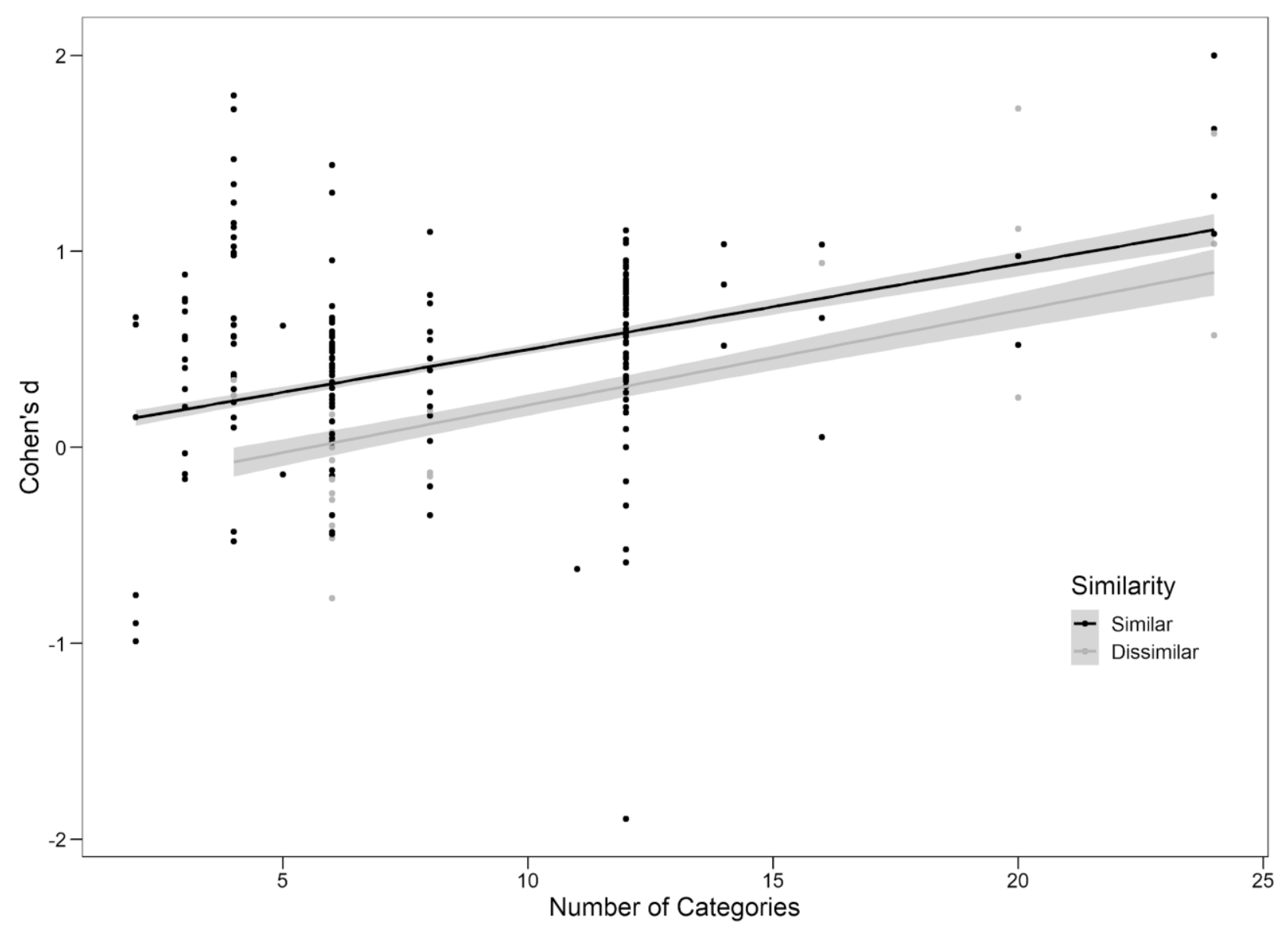




\section{Figure 4}

Four Examples Illustrating Uneven Distribution of Focal Moderators Across Experiments
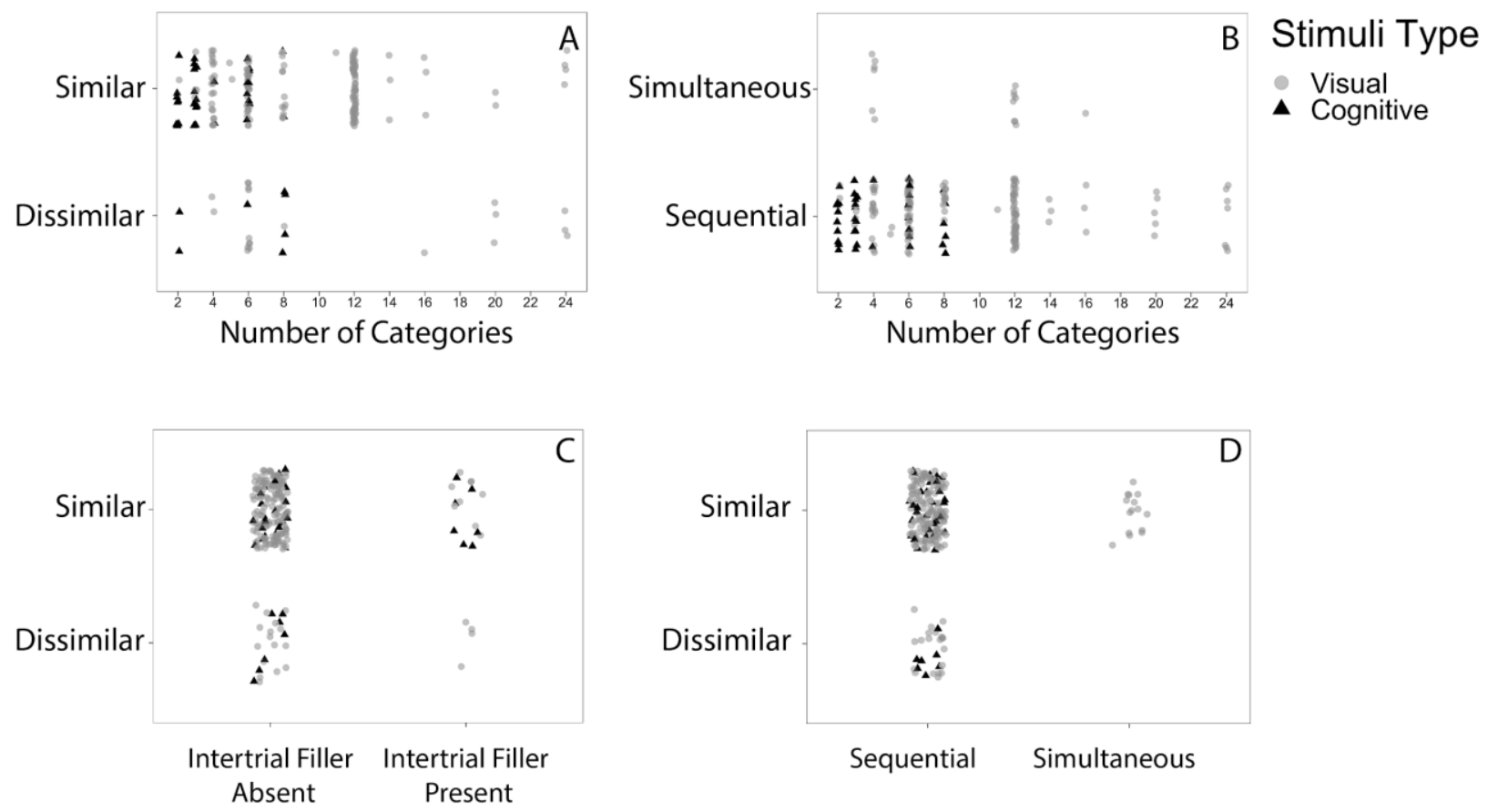

Note. Data points are jittered due to the categorical nature of the moderators. 